\title{
Compact Balanced-to-Balanced Diplexer Based on Split-Ring Resonators Balanced Bandpass Filters
}

\author{
Armando Fernández-Prieto $^{\circledR}$, Member, IEEE, Aintzane Lujambio, Ferran Martín, Fellow, IEEE, \\ Jesús Martel, Senior Member, IEEE, Francisco Medina ${ }^{\circledR}$, Fellow, IEEE, \\ and Rafael R. Boix, Member, IEEE
}

\begin{abstract}
A compact balanced-to-balanced diplexer composed of two balanced bandpass filters is proposed in this letter. The balanced filters are implemented using compact edge-coupled square split-ring resonators. The design methodology is based on the standard coupled-resonators filter synthesis procedure. First, each filter is independently designed. Then, they are connected to a common differential input port in order to achieve the desired diplexing operation, with the pertinent adjustments to take into account the loading effect of the second filter. Magnetic coupling inherently prevents common-mode transmission. An illustrative prototype example is provided with simulations and measurements to demonstrate the benefits of the proposed topology.
\end{abstract}

Index Terms-Balanced-to-balanced (B-B) diplexer, inherent common-mode (CM) rejection, magnetic coupling, split-ring resonators (SRRs).

\section{INTRODUCTION}

$\mathbf{T}$ HE interest in balanced/differential circuits has considerably increased along the last couple of decades [1]. In comparison with single-ended signals, differential signals offer enhanced electrical performance in terms of signalto-noise ratio, noise immunity, crosstalk, and electromagnetic interference. In recent years, with the requirement for multiband services, differential diplexers have attracted the interest of the microwave community [2]-[7]. A welldesigned balanced-to-balanced (B-B) diplexer must simultaneously provide good differential-mode (DM) performance, strong common-mode (CM) rejection, and high isolation between the output ports. Several strategies have been proposed to achieve all those goals. For example, resonators with different DM and CM resonance frequencies are used

Manuscript received November 9, 2017; accepted January 13, 2018. Date of publication February 6, 2018; date of current version March 9, 2018. This work was supported by the Spanish Ministerio de Economía y Competitividad with European Union FEDER Funds under Contract TEC2017-84724-P and Contract TEC2016-75650-R, in part by the Spanish Junta de Andalucía under Project P12-TIC-1435, and in part by the Generalitat de Catalunya under contract 2014SGR-157. (Corresponding author: Armando Fernández-Prieto.)

A. Fernández-Prieto, F. Medina, and R. R. Boix are with the Departamento de Electrónica y Electromagnetismo, Facultad de Física, Universidad de Seville, 41012 Seville, Spain (e-mail: armandof@us.es).

A. Lujambio is with the Parts Laboratory Department, Alter Technology TÜV Nord S.A.U., 41092 Seville, Spain.

F. Martín is with the CIMITEC, Departament d'Enginyeria Electrònica, Universitat Autònoma de Barcelona, 08193 Bellaterra, Spain.

J. Martel is with the Departamento de Física Aplicada II, Escuela Técnica Superior de Arquitectura, 41012 Seville, Spain.

Color versions of one or more of the figures in this paper are available online at http://ieeexplore.ieee.org.

Digital Object Identifier 10.1109/LMWC.2018.2794824 in [2] and [3]. The same approach is considered in [4], with the novelty of including via holes in order to connect the resonators to ground. CM rejection is sacrificed in [5] by introducing mutual couplings between stub-loaded inputoutput lines in order to improve DM out-of-band performance. A modification of [5] is proposed in [6] to improve the CM rejection. The previous proposals provide good DM and CM performance at the expense of using complicated geometries and/or the presence of via holes. Hybrid microstrip/slot-line resonators are used in [7], although this approach has some practical limitations since, in many situations, the integrity of the ground plane must be preserved. In this letter, a compact $\mathrm{B}-\mathrm{B}$ diplexer is proposed. The device is designed by using two different balanced bandpass filters (BBPFs) based on magnetically coupled edge-coupled square split-ring resonators (EC-SRRs). The use of magnetic coupling reduces the $\mathrm{CM}$ transmission [8], and the use of EC-SRRs leads to a very compact design.

\section{Proposed BAlanced Diplexer}

\section{A. Design Methodology}

The implementation of the B-B diplexer in this letter starts with the design of the BBPFs for each balanced output port (or channel) (22 and 33 in Fig. 1). Each filter, being composed of two magnetically coupled EC-SRRs, is independently designed. The reasons behind the choice of EC-SRRs and magnetic coupling are: 1) EC-SRRs, consisting of a pair of tightly coupled concentric metallic split rings, are electrically small resonators yielding a higher level of compactness than other alternative printed resonators [9], [10] and 2) the CM transmission is reduced over a wide frequency range thanks to the use of magnetic coupling [8]. For the filters in Fig. 1, the coupled-resonators design procedure described in [11] has been used. Such method makes use of the external quality factors, $Q_{e}$, and the coupling coefficients, $M$. For given DM filter specifications, both parameters can be theoretically calculated by means of the following expressions

$$
\begin{aligned}
M_{i, i+1} & =\frac{\Delta}{\sqrt{g_{i} g_{i+1}}}, \quad \text { for } i=1, \ldots, n-1 \\
Q_{e 1} & =\frac{g_{0} g_{1}}{\Delta} \quad Q_{\mathrm{en}}=\frac{g_{n} g_{n+1}}{\Delta}
\end{aligned}
$$

where $n$ is the filter order, $\Delta$ is the fractional bandwidth, and $g_{j}(j=1, \ldots, n+1)$ are the low-pass prototype element values for the desired filter response. For the following specifications: Butterworth response, $n=2$, fractional bandwidth $\Delta^{l}=11.5 \%$ and $\Delta^{u}=7.2 \%$, and center frequencies 


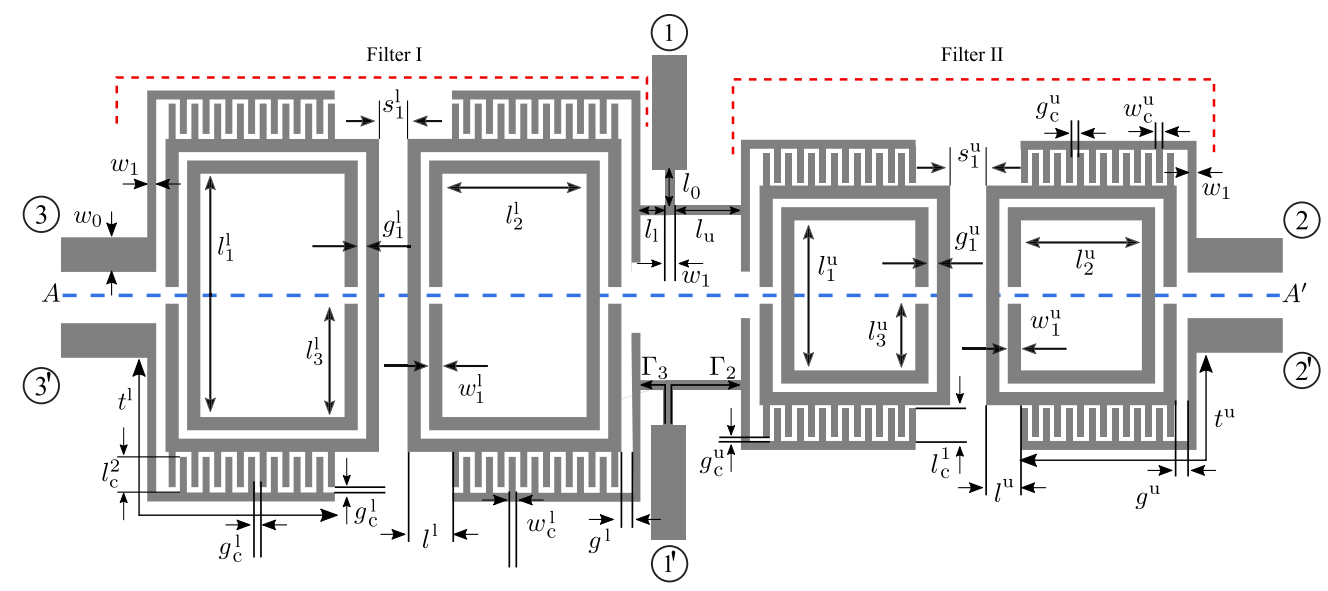

Fig. 1. Layout of the proposed balanced diplexer (not to scale). Dimensions in mm are: $w_{0}=2.54, w_{1}=w_{c}^{l}=g_{c}^{l}=g_{1}^{l}=0.2, g^{l}=0.4, w_{1}^{l}=0.3$, $l_{c}^{2}=1.3, l^{l}=2.8, l_{1}^{l}=12.7, l_{2}^{l}=8, l_{3}^{l}=6.2, s_{1}^{l}=0.9, t^{l}=11.96, w_{c}^{u}=g_{c}^{u}=g_{1}^{u}=0.2, g^{u}=0.4, w_{1}^{u}=0.3, l_{c}^{1}=1, l^{u}=2, l_{1}^{u}=7.7, l_{2}^{u}=6, l_{3}^{u}=3.7$, $s_{1}^{u}=1.1, t^{u}=8.46, l_{l}=1.47, l_{u}=3.2$, and $l_{0}=3.2$.

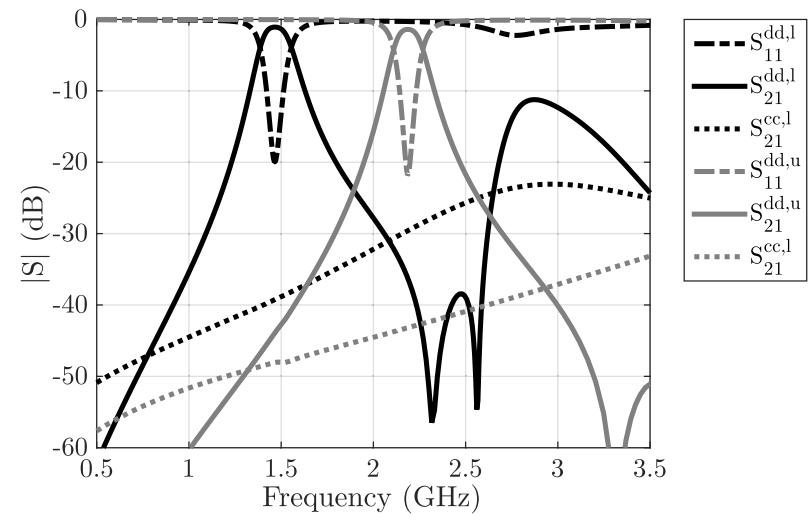

Fig. 2. Simulated (electromagnetic) DM and CM responses for the designed lower and upper BBPFs. Simulations have been carried out with $A D S$ Momentum software.

$f_{0 d}^{l}=1.5 \mathrm{GHz}$ and $f_{0 d}^{u}=2.2 \mathrm{GHz}$, the theoretical values for the $M$ 's and $Q$ 's calculated from (1) and (2) are: $M_{1,2}^{l}=0.081, M_{1,2}^{u}=0.051, Q_{e 1}^{l}=Q_{e 2}^{l}=Q_{e}^{l}=12.29$, and $Q_{e 1}^{u}=Q_{e 2}^{u}=Q_{e}^{u}=19.64$. The superscripts " $l$ " and " $u$ " stand for the lower $(l)$ and upper $(u)$ differential passbands (channels 33 and 22 in Fig. 1). The parameters of the used substrate are $\varepsilon_{r}=3.0$, thickness $h=1.016 \mathrm{~mm}$, and $\tan \delta=0.0022$. The filters are independently designed to fulfill the theoretical requirements for $Q_{e}$ and $M_{1,2}$. Design curves similar to those reported in [8] have been generated (not included here to save space). Note that interdigital capacitors have to be used in order to achieve the desired value of $Q_{e}$ [8]. The simulated responses for both filters are shown in Fig. 2. The two transmission zeros (TZs) in the upper stopband of each channel filter are an intrinsic feature of the EC-SRR structure. They correspond to those frequencies at which the even or odd electrical lengths of the resonators is $\pi$. Good DM performance and a high CM rejection can be observed in both differential passbands. The diplexing operation can be now obtained by joining the filters to a common differential input port (see Fig. 1). The key point in designing the T-junction that connects both filters is that the external quality factors at the filters inputs must be those imposed by the design specifications. This means that the $\mathrm{T}$-junction must be designed accounting for the loading effect of the accompanying filter so as to preserve the required external $Q$ 's, thus ensuring low-return loss level at both output channels, i.e., $\left|\Gamma_{3}\right| \approx 0$ at $f_{0 d}^{l}=1.5 \mathrm{GHz}$ while $\left|\Gamma_{2}\right| \approx 0$ at $f_{0 d}^{u}=2.2 \mathrm{GHz}$ [3]. We have proceeded as follows: 1) the T-junction with arbitrarily chosen values of $w_{1}, l_{0}, l_{l}$, and $l_{u}$ has been introduced; 2) the lengths of the branch feeding lines, $l_{l}$ and $l_{u}$, have been used as adjustable parameters to fit the required external quality factors at the filters input ports such that $Q_{e 1}^{l}=Q_{e 1}^{l}=12.29$ and $Q_{e 1}^{u}=Q_{e 1}^{u}=19.64$ (i.e., two physical parameters for two electrical parameters). The final dimensions of the proposed B-B diplexer are detailed in Fig. 1.

\section{B. Experimental Results}

In order to validate our proposal, the B-B diplexer in Fig. 1 has been fabricated and measured using the Agilent PNA-N5221A four-port network analyzer. Simulated and measured DM and CM responses are shown in Fig. 3(a) and (b), where a photograph of the fabricated prototype has been included.

Good agreement between simulations and measurements can be observed. Measured results exhibit passband center frequencies at 1.47 and $2.19 \mathrm{GHz}$, with the fractional bandwidths of $11.5 \%$ and $7 \%$ and the insertion loss level of 0.94 and $2.2 \mathrm{~dB}$, respectively. The experimental DM isolation (Iso) is better than $40 \mathrm{~dB}$ for the lower band and about $40 \mathrm{~dB}$ for the upper band. In addition, the measured $\mathrm{CM}$ rejection is about 40 and $45 \mathrm{~dB}$ for the lower- and upper-band channels, respectively. Note that a third TZ appears in the transmission response when the two differential filters are assembled to build up the diplexer. This is a natural consequence of the imposed design goals of the T-junction.

A comparison with previous contributions is included in Table I. Table I demonstrates that the proposed structure provides the most compact design, thanks to the use of EC-SRRs to implement the filters by halving the area of the smallest previous contributions. In addition, in terms of common-mode rejection ratio, the proposed diplexer is found to be very competitive. Moreover, the high DM isolation is 
TABLE I

Comparison Between Balanced and Balun Diplexers Based on Coupled Resonators

\begin{tabular}{|c|c|c|c|c|c|c|c|}
\hline & \multirow{2}{*}{ Type } & \multirow{2}{*}{$\begin{array}{c}\text { Area } \\
\end{array}$} & & \multicolumn{5}{|c|}{ Differential-mode } & Common-mode \\
\cline { 5 - 8 } & & $\lambda_{g}^{2} \dagger \dagger$ & $f_{0 d}^{1, \mathrm{u}}(\mathrm{GHz})$ & $3-\mathrm{dB} \Delta^{1, \mathrm{u}}(\%)$ & $\mathrm{IL} f_{0 d}^{1, \mathrm{u}}(\mathrm{dB})$ & Iso $(\mathrm{dB})$ & CMRR @ $f_{0 d}^{1, \mathrm{u}}(\mathrm{dB})$ \\
\hline \hline$[2]$ & U-B & 0.315 & $1 / 1.2$ & $10.5 / 10.4$ & $2.2 / 2.35$ & $46.5 / 46.5$ & $55 / 50$ \\
\hline$[3]$ & U-B & 0.202 & $1.847 / 2.467$ & $11.6 / 8.7$ & $1.48 / 1.78$ & $\approx 45 / 45$ & $38.5 / 38.22$ \\
\hline$[4]$ & U-B & 0.225 & $1.93 / 2.46$ & $7.2 / 4.5$ & $0.67 / 1.07$ & $42.1 / 39.5$ & $36.7 / 42.9$ \\
\hline$[4]$ & B-B & 0.225 & $1.94 / 2.46$ & $6.7 / 4.5$ & $0.88 / 0.98$ & $42.1 / 40.1$ & $26.4 / 46.9$ \\
\hline$[7]$ & U-B & 0.544 & $2.41 / 3.57$ & $4.6 / 8.7$ & $1.56 / 1.66$ & $41.3 / 44.5$ & $55.7 / 53.6$ \\
\hline$[7]$ & B-B & 0.550 & $2.45 / 3.55$ & $6.7 / 8.2$ & $1.95 / 2.11$ & $39.5 / 44.5$ & $50.2 / 47.7$ \\
\hline$[5]$ & B-B & 0.099 & $2.46 / 3.65$ & $8.1 / 4.9$ & $1.5 / 2$ & $33 / 42$ & $28.5 / 30$ \\
\hline$[6]$ & B-B & N/A & $2.45 / 3.6$ & $6 / 3$ & $1.3 / 1.8$ & $\approx 35 / 55$ & $\approx 56.7 / 48.2$ \\
\hline Fig. 1 & B-B & $\mathbf{0 . 0 4 6}$ & $1.47 / 2.19$ & $11.5 / 7$ & $0.94 / 2.2$ & $\mathbf{4 3 . 3 / 4 0}$ & $39.06 / 42.8$ \\
\hline
\end{tabular}

$\lambda_{g} \dagger$ : Guided wavelength @ $f_{0 d}^{1}$; U-B*: Unbalanced-to-balanced; N/A: substrate characteristics not provided.
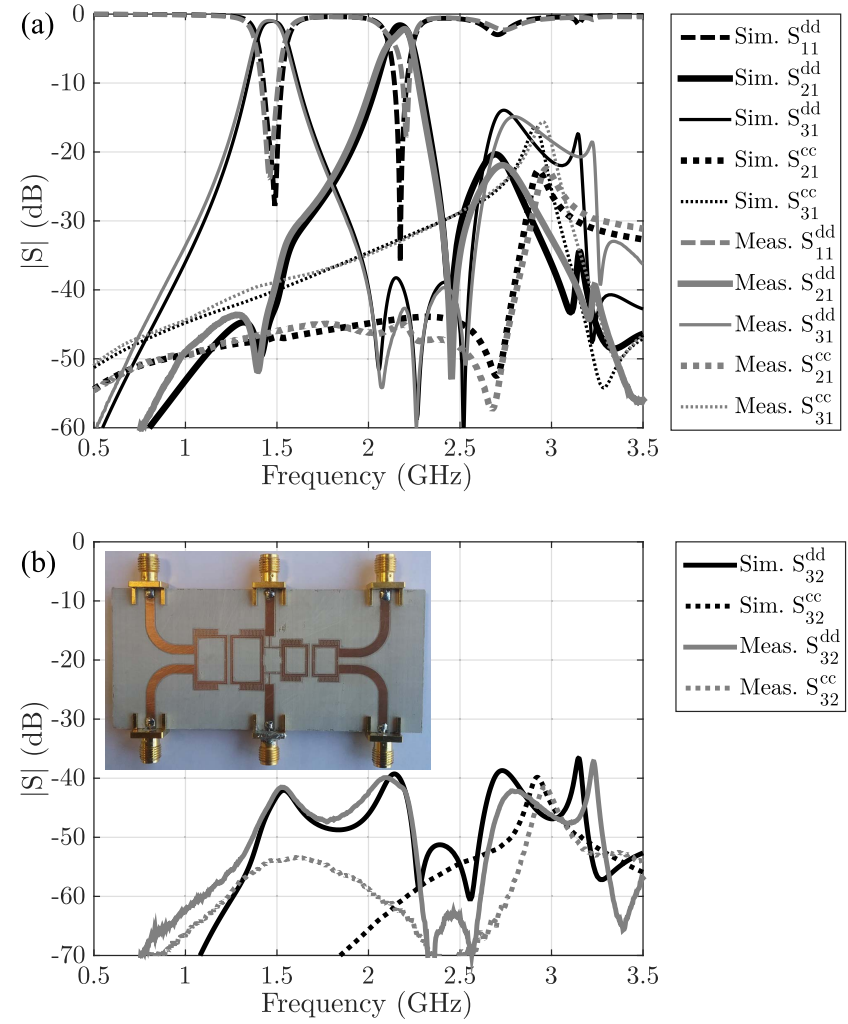

Fig. 3. DM and CM simulated and measured responses (both channels) of the designed diplexer (see Fig. 1). (a) Reflection and transmission. (b) Isolation.

observed in both passbands. These results prove that the use of EC-SRRs with magnetic coupling leads to compact diplexer design with high levels of CM rejection and DM isolation.

\section{CONCLUSION}

A compact B-B diplexer has been presented in this letter. It consists of two balanced bandpass filters based on magnetically coupled EC-SRRs. Both filters have been straightforwardly designed by means of a well-known approach based on the coupling coefficients and external quality factors. High CM rejection for both channels is achieved by virtue of the magnetic coupling between EC-SRRs, providing significant interresonator distance and, hence, efficiently blocking the common mode up to high frequencies. The DM and CM isolations are also very good. Nevertheless, the main competitive advantage of the proposed diplexer is its small size, achieved thanks to the use of EC-SRRs. The lack of vias and defected ground structures are additional beneficial aspects for the application of the proposed diplexer in real scenarios.

\section{REFERENCES}

[1] W. R. Eisenstant, B. Stengel, and B. M. Thompson, Microwave Differential Circuit Design Using Mixed-Mode S-Parameters. Boston, MA, USA: Artech House, 2006.

[2] C.-H. Wu, C.-H. Wang, and C.-H. Chen, "A novel balanced-tounbalanced diplexer based on four-port balanced-to-balanced bandpass filter," in Proc. 38th Eur. Microw. Conf., Amsterdam: The Netherlands, 2008, pp. 28-31.

[3] Q. Xue, J. Shi, and J.-X. Chen, "Unbalanced-to-balanced and balancedto-unbalanced diplexer with high selectivity and common-mode suppression," IEEE Trans. Microw. Theory Techn., vol. 59, no. 11, pp. 2848-2855, Nov. 2011.

[4] C.-H. Lee, C.-I. G. Hsu, and P.-H. Wen, "Balanced and balun diplexers designed using center-grounded uniform-impedance resonators," Microw. Opt. Technol. Lett., vol. 56, no. 3, pp. 555-559, Mar. 2014.

[5] Y. Zhou, H.-W. Wei, and Y. Zhao, "Compact balanced-to-balanced microstrip diplexer with high isolation and common-mode suppression," IEEE Microw. Wireless Compon. Lett., vol. 24, no. 3, pp. 143-145, Mar. 2014.

[6] H. Deng, Y. Zhao, Y. Fu, Y. He, and X. Zhao, "High selectivity and $\mathrm{CM}$ suppression microstrip balanced BPF and balanced-to-balanced diplexer," J. Electromagn. Waves Appl., vol. 27, no. 8, pp. 1047-1058, May 2013.

[7] P.-H. Weng, C.-I. G. Hsu, C.-H. Lee, and H.-H. Chen, "Design of balanced and balun diplexers using stepped-impedance slot-line resonators,' J. Electromagn. Waves Appl., vol. 28, no. 6, pp. 700-715, Feb. 2014.

[8] A. Fernández-Prieto, A. Lujambio, J. Martel, F. Medina, F. Mesa, and R. R. Boix, "Simple and compact balanced bandpass filters based on magnetically coupled resonators," IEEE Trans. Microw. Theory Techn., vol. 63, no. 6, pp. 1843-1853, Jun. 2015.

[9] R. Marqués, F. Martín, and M. Sorolla, Metamaterials With Negative Parameters: Theory, Design and Microwave Applications. Hoboken, NJ, USA: Wiley, 2007.

[10] J. García-García, J. Bonache, I. Gil, F. Martín, M. C. Velázquez-Ahumada, and J. Martel, "Miniaturized microstrip and CPW filters using coupled metamaterial resonators," IEEE Trans. Microw. Theory Techn., vol. 54, no. 6, pp. 2628-2635, Jun. 2006.

[11] J.-S. Hong, Microstrip Filters for RF/Microwave Applications. 2nd ed. New York, Ny, USA: Wiley 2011, ch. 7. 